LBL- -29956

DE91 007745

\title{
Hole Coupling Resonator for Free Electron Lasers*
}

\section{Ming Xie and Kwang-Je Kim}

\author{
Lawrence Berkeley Laboratory \\ University of Califomia \\ Berkeley, CA 94720, USA
}

Paper Presented at Twelfth International Free Electron Laser Conference

Paris, France, September 17-21, 1990

* This work was supported by the Director, Office of Energy' Research, Office of Basic Energy

Sciences, Materials Sciences Division, of the U.S. Department of Energy under contract No. DEAC03-76SF00098.

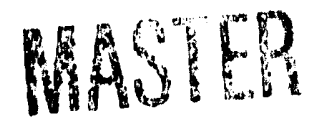




\begin{abstract}
The performance of two mirror resonators with holes for output coupling is studied for free electron laser application using a Fox-Li type code. The mode profiles inside and ou ide the cavity, the diffraction losses at the mirror edges and apertures, the amount of useful power coupled through the hole, e.t.c., are calculated for the dominant mode for different hole and mirror dimensions. It is found that resonators in near concentric geometry can develop a mode degeneracy in certain cases, which should be avoided for the stability of the free electron laser gain and output. A resonator configuration for a free electron laser at Lawrence Berkeley Laboratory was found which can provide satisfactory performance over a wavelength range from 25 to 50 microns. The possibility of further increasing the tuning range by an adjustable intracavity aperture is discussed.
\end{abstract}

\title{
1. Introduction
}

An infrared free electron laser (FEL) operating between 3 and 50 microns is being designed for the Chemical Dynamics Research Laboratory (CDRL) at Lawrence Berkeley Laboratory [1]. In search for efficient, broadly tunable optical output coupling schemes for the CDRL-FEL, we are investigating resonators with a circular hole in the center of one of the end mirrors. This paper is a summary of our study so far on this subject.

Hole coupling has been proven to be effective in the far-infrared region where dielectric mirrors are not available. In the 1960s Patel et al [2] used the hole coupling for several lines in Ne and in Ne-He gas lases's ranging from 32 to 57 microns. More recently hole coupling was used for the LANL FEL experiment at 10 microns [3]. Even in the near infrared region, where dielectric mirrors are available, the hole coupling based on metal mirrors could be superior due to its broader tuning range and higher damage threshold. Other potentially attractive coupling schemes using metal surfaces are that based on an unstable resonator [4] and that based on the intracavity 
gratings [5].

Several resonator configurations have been studied for hole coupling silice Patel's early experiment. Confocal resonator was analyzed by McCumber [6] and Shennagel [7] using a perturbation technique, and by McNice and Derr [8] and Moran [9] using Fox-Li method. FabryPerot resonator was treated by $\mathrm{Li}$ and Zucker [10] and Yoshida et al [11]. Some nonconfocal resonators was investigated by Tsuji et al [12]. Pantell et al [13] presented an approximate analysis of FEL resonators with holes in the mirrors, where the holes are for the electron beam passage rather than for the coupling of the optical beam.

We have developed a new, Fox-Li type code for axially symmetric resonators with holes and an arbitrary set of intracavity apertures. In our numerical study we pay close attention to the issues important for FEL operation in a user facility: The intracavity mode profile should have a good overlap with the electron beam for efficient energy extraction; A sufficient fraction of the energy should be coupled out through the hole; The out coupled mode should be close to the free space Gaussian mode for an efficient transport to the experimental station; A single cavity configuration should cover as wide a wavelength range as possible to avoid a frequent change of sensitive optical components.

In studying the resonators for the CDRL-FEL, it is found that the behavior of a hole coupling resonator in the near concentric geometry is somewhat different from those in the other stable configurations. Thus the mode profile across the hole remains more or less uniform, ensuring a good output coupling. Also, the mode can develop a degeneracy at a certain hole size. Avoiding the mode degeneracy is an important consideration for the design of a hole coupling resonator.

The analysis in this paper is approximate in the sense that the effect of the FEL interaction 
on the cavity mode has not been taken into account. Such effect is expected to be small when the saturated gain (which is equal to the total round trip loss) is small, as are the cases studied here.

\section{Calculation of the Hole Coupling Resonator Performance}

A new code, named HOLD (HOLe coupling and Diffraction), calculates the dominant axially symmetric eigenmode in a resonator with two perfectly reflecting circular mirrors of finite size. Each mirror may have a circular hole in the center. In addition, any number of circular apertures may be placed at any locations along the optical axis inside cavity. The mode propagation in each round trip is calculated via the Fresnel-Kirchhoff integral transform [14]. A dominant eigenmode is obtained by iterating the round-trip propagation, starting from a noisy distribution in phase and amplitude at one mirror surface. The loss at each element inside cavity is also calculated.

The nominal configuration of the FEL resonator studied in this paper is shown schematically in Fig.1. The effect of the undulator bore is approximately taken into account by two apertures at each end of the undulator, the aperture radius chosen to be the same as that of the bore. We expect the approximation to be adequate provided the loss at the undulator bore is small. However, we have not studied in detail the validity of the approximation for the cases studied here, for which the loss at the apertures can be up to $10 \%$. The resonator for the CDRL-FEL is in the near concentric configuration, with the parameters listed in Table 1.

An cxample of the transverse intensity profile of an eigenmode calculated by the code is shown in Fig. 2 at several locations along the optical axis. The right mirror located at the upperright corner in the graph is the one with a hole. The hole edge is marked by an arrow. Notice that a complete description of the mode profile requires a specification of the wave profile during one round trip, and that the wave profile travelling in one direction is in general different from that in the opposite direction. In the figure, the dashed lines are the profiles of the wave travelling from 
the right mirror toward the left while the solid lines are for the wave in the opposite direction. Notice the characteristic diffraction pattern from the edge of the hole in the wave moving away from the hole, for example, the Arago's spot appearing as a narrow peak at short distances from the hole [15]. The location and the edge of the two apertures are indicated by the two horizontal arrows in the graph.

Our procedure of finding the eigenmode by iterating a noisy initial field will lead to the dominant eigenmode, which is defined to be the mode with the lowest round trip loss. When the hole radius is small, the dominant mode is also the fundamental mode, which can be loosely defined as the mode having the simplest structure, with its energy mainly concentrated in the mirror center. As the hole becomes larger, the mode structure becomes more complicated. For a confocal resonator, which has been extensively studied in the literature, the change of the mode profile as a function of the hole size is continuous [8]. However, for the case of the near concentric resonator, we have observed a discontinuous change in the mode profile. This is caused by the mode degeneracy, i.e., the fact that the loss of the fundamental mode increases as the hole becomes larger and at some point becones the same as that of the next higher order mode. Beyond this point, the dominant mode is the next higher order mode. Therefore the mode changes discontinuously from the fundamental mode to the higher order mode as the hole radius passes through the value corresponding to the degeneracy. At the point of the degeneracy, the mode is a linear superposition of the fundamental and the higher order mode with the coefficients determined by the initial conditions. The mode degeneracy here is similar to that found in the unstable resonator [15]. In fact, we conjecture that a near concentric resonator with holes becomes effectively an unstable resonator due to the additional focusing by the holes.

In Fig.3, the total fractional power loss per round trip, the hole coupling efficiency defined to be the ratio of the useful loss through the hole to the total loss [8] and the number of the iteration required for the convergence are shown as a function of the hole radius. The evidence of mode 
degeneracy is clearly seen at hole radius $=4 \mathrm{~mm}$; Although the total loss is continuous, the coupling efficiency changes in a discontinuous step across this point. Also, notice that the number of iterations required for convergence becomes very large at the degenerate point. This is because the profile switches back and forth between two modes indefinitely at the degenerate point. The mode profile for hole radius less than $4 \mathrm{~mm}$ is of the general shape shown in Fig.2, while that for hole radius larger than $4 \mathrm{~mm}$ is shown in Fig.4. That the latter belongs to a higher order mode may be inferred from the presence of a minima in the profile at mirror locations.

Notice that, in either Fig. 2 or Fig.4, the profile of the wave approaching the mirror is more or less uniform across the coupling hole, implying that a reasonable amount of intracavity power is coupled out through the hole. This behavior is in contrast to the tendency of the mode in the stable, non-concentric resonators to develop a null at the location of the holes [13]

\section{Optimization of the Hole Coupling Resonator for CDRL-FEL}

Several considerations enter into the optimization of a hole coupling resonator for FELs: The first is regarding the total fractional power loss in a round-trip. The optimum value for the total loss depends on factors such as small signal gain and saturated extraction efficiency. For the CDRL-FEL we require the loss to be roughly in the range from 10 to 20 percent.

We expect a smooth, singly peaked profile in the interaction region to have a better overlap with electron beams than a multiply peaked one. Thus we require the mode profile in the direction of the electron beam to be of a smooth, Gaussian like shape. The profile of the fundamental mode will satisfy this requirement.

It is clear that the mode degeneracy discussed in the previous section should be avoided; The beating of two degenerate modes causes the overall field distribution to oscillate, sometimes 
violently, leading to a corresponding gain modulation. Also, the number of passes required for the convergence should be less than that required for FEL saturation.

It follows from these considerations that the hole radius should be chosen to be smaller than that corresponding to the mode degeneracy. The value of the hole radius can be determined from Fig.3. if the desired total round trip loss is known. From this figure, we see that the hole coupling efficiency is about $50 \%$ in the region dominated by the fundamental mode.

Whether the out coupled beam can be efficiently transported to a user station is another important factor in the design of a coupling scheme. Since the fundamental Gaussian mode in free space can usually be efficiently transported, it is of interest to know what fraction of the out coupled optical energy is contained in the fundamental Gaussian mode in the Laguerre-Gaussian expansion. (This expansion contains two free parameters, the mode waist and the Rayleigh range. These parameters are chosen so that the power in the fundamental Gaussian mode is maximized.) With this in mind, we define the Gaussian quality factor for the out coupled mode as the ratio of the power in the fundamental Gaussian mode to the total power. In the special case when the field distribution is uniform over the hole area (the far field diffraction pattern in that case is known as the Airy pattern), the Gaussian quality factor is $81 \%$. We find that the Gaussian quality factor is close to this value when the hole radius is below the mode degeneracy point.

To illustrate the tuning range, the performances of the hole coupling resonator at two wavelengths, 25 and 50 microns, for the CDRL-FEL are shown superposed in Fig.5. We see that, for hole radius $=3 \mathrm{~mm}$, the loss varies from $20 \%$ to $15 \%$ and the coupling efficiency from $47 \%$ to $38 \%$ as the wavelength changes from 25 to 50 microns. The Gaussian quality factor is close to $81 \%$ in all cases. Also the mode discrimination and intracavity mode quality are good for all wavelength within the range because the mode degeneracy for a longer wavelength occurs at a larger hole. Thus the resonator with a coupling hole of radius $=3 \mathrm{~mm}$ satisfies all the requirements 
in the wavelength range from 25 to 50 microns.

From this example, a general procedure to determine the parameters of a hole coupling resonator with a broad wavelength tuning may be outlined as follows:

1. Starting from the short wavelength end of the desired tuning range, choose a hole size smaller than but close to the first degenerate point. This will guaranty the fundamental mode operation and the Gaussian quality factor close to $80 \%$ at longer wavelength.

2. Fine tune the hole size until all requirements are satisfied at the short wavelength end.

3. Fix the hole size at the value determined in step 2 and increase the wavelength until either total loss or coupling efficiency drops below the required level. The longer wavelength end of the tuning range is then determined.

Finally, it should be mentioned that the apertures play an important role in the optimization described in the above. Figure 6 shows the mode profile for the same resonator as for Fig. 2 but with the two apertures removed. The intracavity apertures introduce more loss to higher order modes implying that the hole size corresponding to the mode degeneracy can be adjusted by adjusting the aperture. Thus the operating wavelength range of a hole coupling resonator can be further increased by placing an adjustable aperture at an appropriate location in the cavity.

\section{Conclusion}

We have shown that the hole coupling resonator can provide satisfactory performance for the CDRL-FEL over a factor of two coverage in wavelengths. A list of performance requirements is outlined and the design strategy for optimum resonator configuration is proposed. Our study indicates the possibility of extending the achievable tuning range by introducing adjustable intracavity apertures. In future work we will include axially asymmetrical mode in our study and 
investigate the effects of gain medium on the resonator performance.

\section{Acknowledgements}

The authors would like to thank Erik Bollt for his contribution in the early stage of developing the simulation code and Andy Kung for helpful discussions.

\section{Feferences}

[1] K.-J. Kim, M. Berz, S. Chattopadhyay, J. Edighoffer, R. Gough, C. Kim, A. H. Kung, W. Stein and M. Xie, "Design Overview of a Highly Stable Infrared Free Electron Laser at LBL", these proceedings.

[2] C. K. N. Patel, W. L. Faust, R. A. McFarlane, C. G. B. Garrett, Applied Physics Letters, Vol-4, No.1,1964, p18.

[3] D. W. Feldman, H. Takeda, R. W. Warren, J. E. Sollid, W. E. Stein, W. J. Johnson, A. H. Lumpkin and R. B. Feldman, NIM, A285, 1989, p11.

[4] A.E. Siegman, private communications.

[5] J.E. Sollid, et. al., Nucl. Instr. Meth., A 285 ( 1989) 147; K.-J. Kim and M. Xie, "Stability and Performance of CDRL-FEL", these proceedings.

[6] D. E. McCumber, B. S. T. J., Vol-44, No.2, 1965, p333, B. S. T. J., Vol-48, No.7, 1969, p1919.

[7] G. Shennagel, Soviet Physics-Technical Physics, Vol-14, No.11, 1970, p1553.

[8] G. T. McNice, V. E. Derr, IEEE Journal of Quantum Electronics, Vol-5, No.12, 1969, p569.

[9] J. M. Moran, IEEE Journal of Quantum Electronics, Vol-6, No.2, 1970, p93.

[10] T. Li, H. Zucker, Journal of the Optical Society of America, Vol-57, No.8, 1967, p984.

[11] Y. Yoshida, H. Ogura, J. Ikenoue, Japanese Journal of Applied Physics, Vol-10, No.6, 1 971, p754. 
[12] M. Tsuji, H. Shigesawa, K. Takiyama, Applied Optics, Vol-18, No.9, 197?, p1334.

[13] R. H. Pantell, J. Feinstein and A. H. Ho, Presented at the eleventh international FEL conference, Naples, Florida, Sept.,1989.

[14] A. G .Fox, T. Li, B. S. T. J. Vol-40, No.3, 1961, p453.

[15] A. E. Siegman, Lasers, University Science Books, 1986.

\section{Caption}

Figure 1. Cross-sectional view of the cylindrical resonator consisting of two circular mirrors and two circular apertures. A circular hole is placed at the center of the right mirror.

Figure 2. Intracavity intensity profiles of the dominant mode at 25 micron wavelength and $2.53 \mathrm{~mm}$ hole radius.

Figure 3. Total round-trip power loss, coupling efficiency and the relative number of iteration passes at 25 micron wavelength.

Figure 4. Total round-trip power loss and coupling efficiency at two wavelengths: 25 micron and 50 micron.

Figure 5. Intracavity intensity profiles of the dominant mode at 25 micron wavelength and $4.56 \mathrm{~mm}$ hole radjus.

Figure 6. Intracavity intensity profiles of the dominant mode for the same resonator as for Fig. 2 but with the two apertures removed. 
Table 1. Resonator parameter for CDRL IRFEL

Cavity Length

Undulator Length ( Aperture Separation )

Mirror Radius of Curvature

Mirror Diameter

Aperture Diameter
8.2 meter

2. meter

4.3 meter

$81 \mathrm{~mm}$

$18.5 \mathrm{~mm}$ 

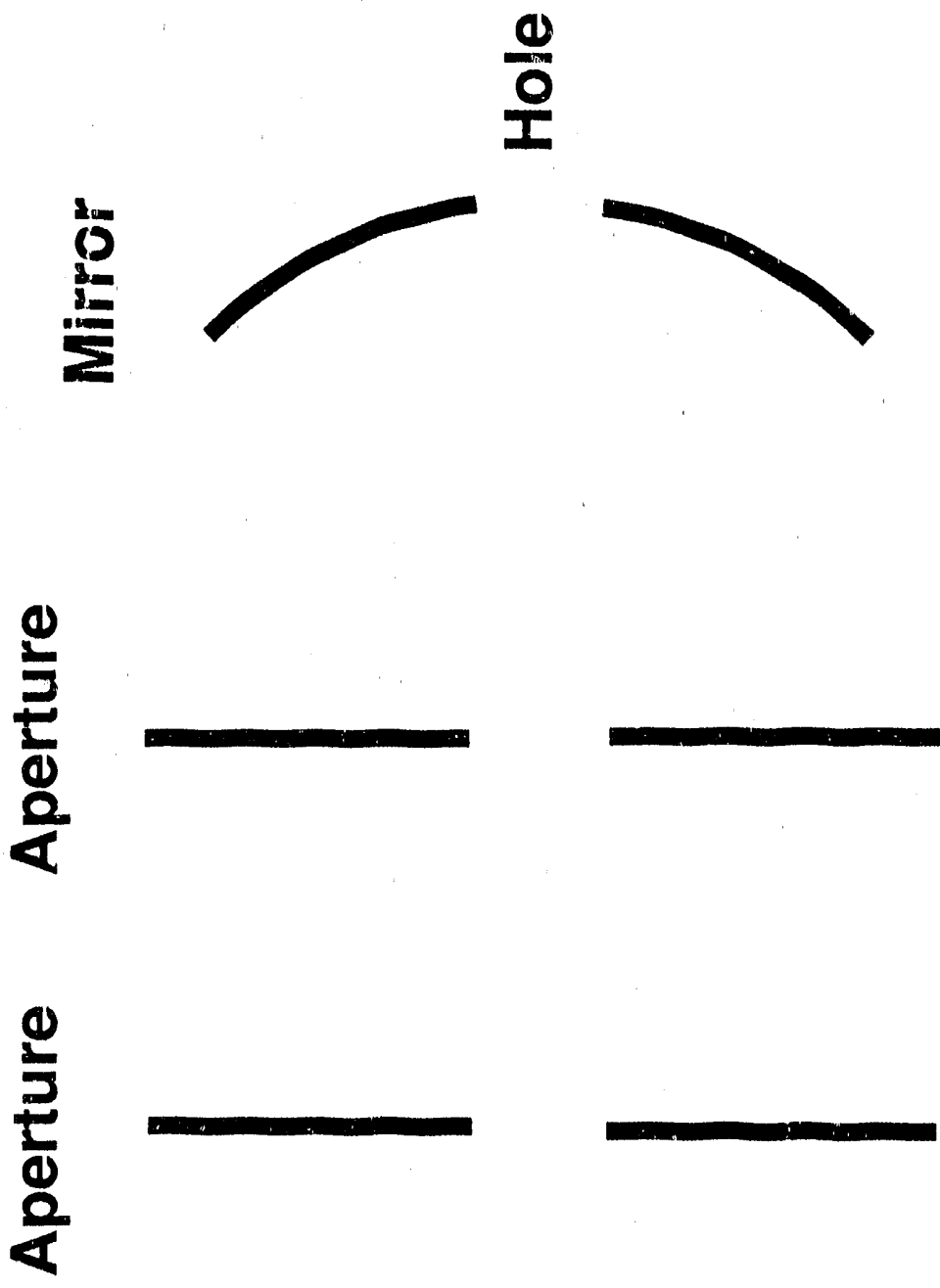

혼

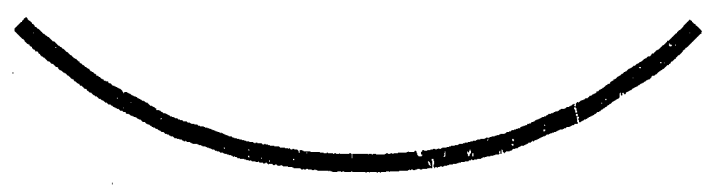




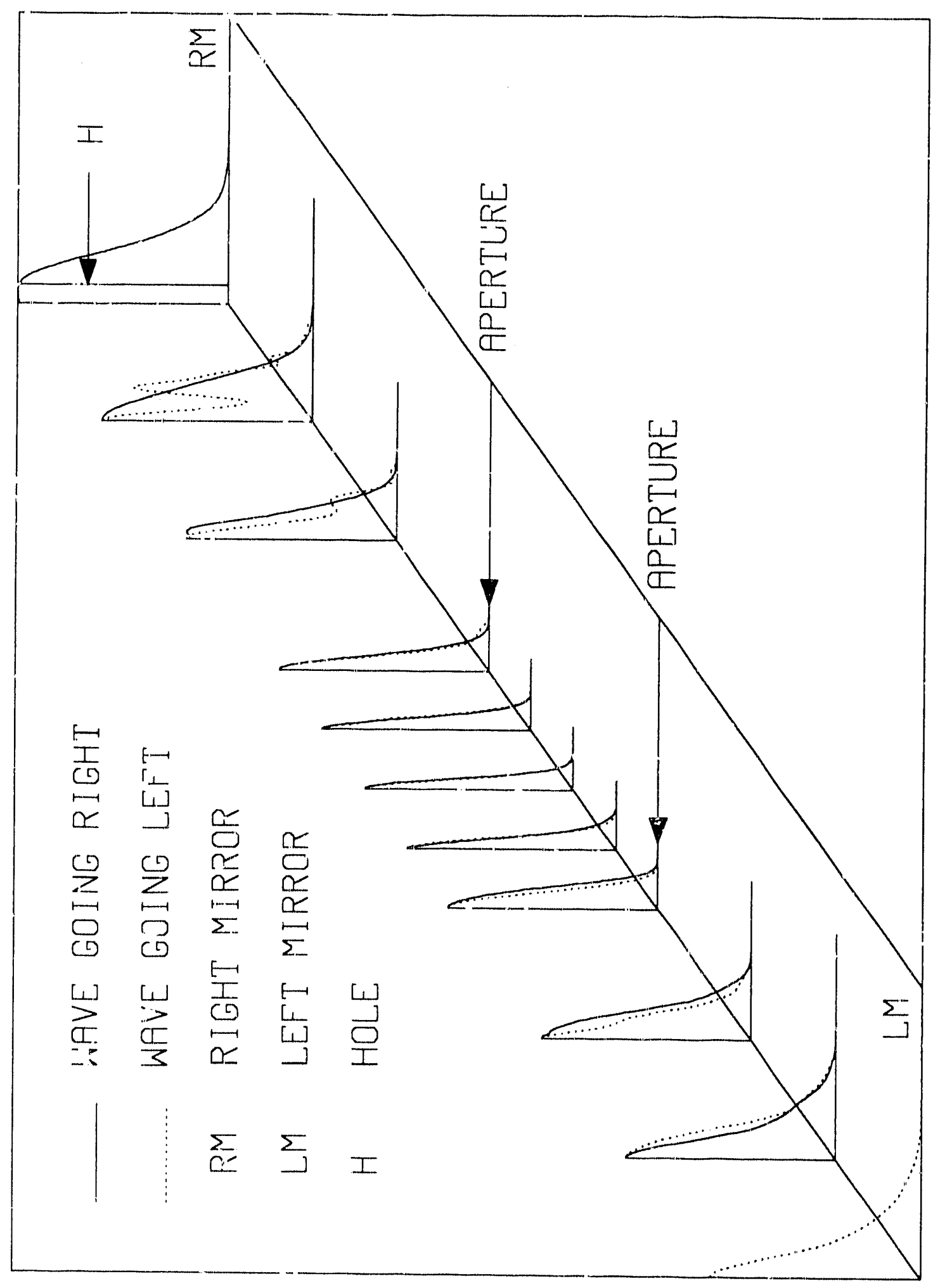

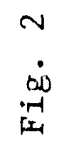




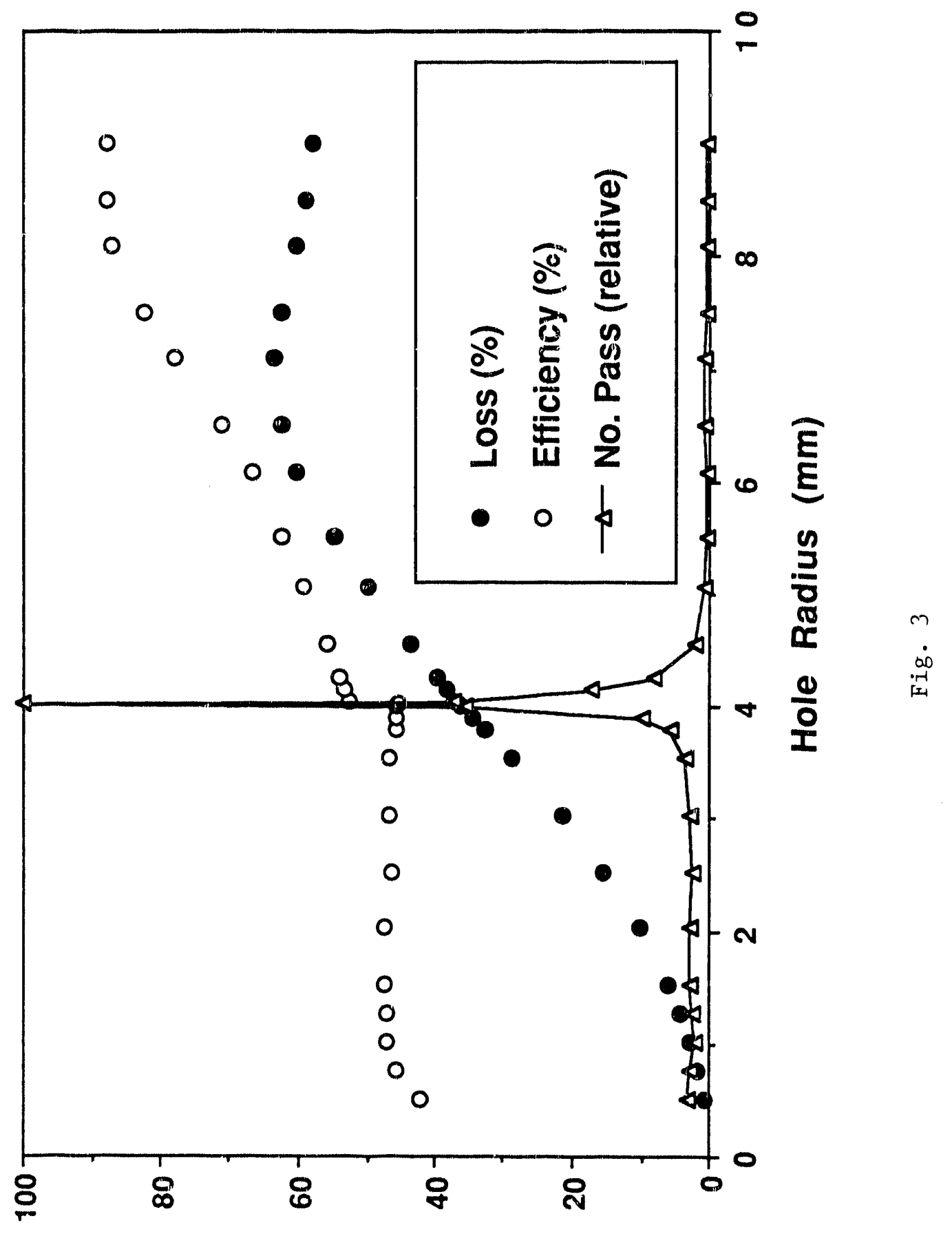




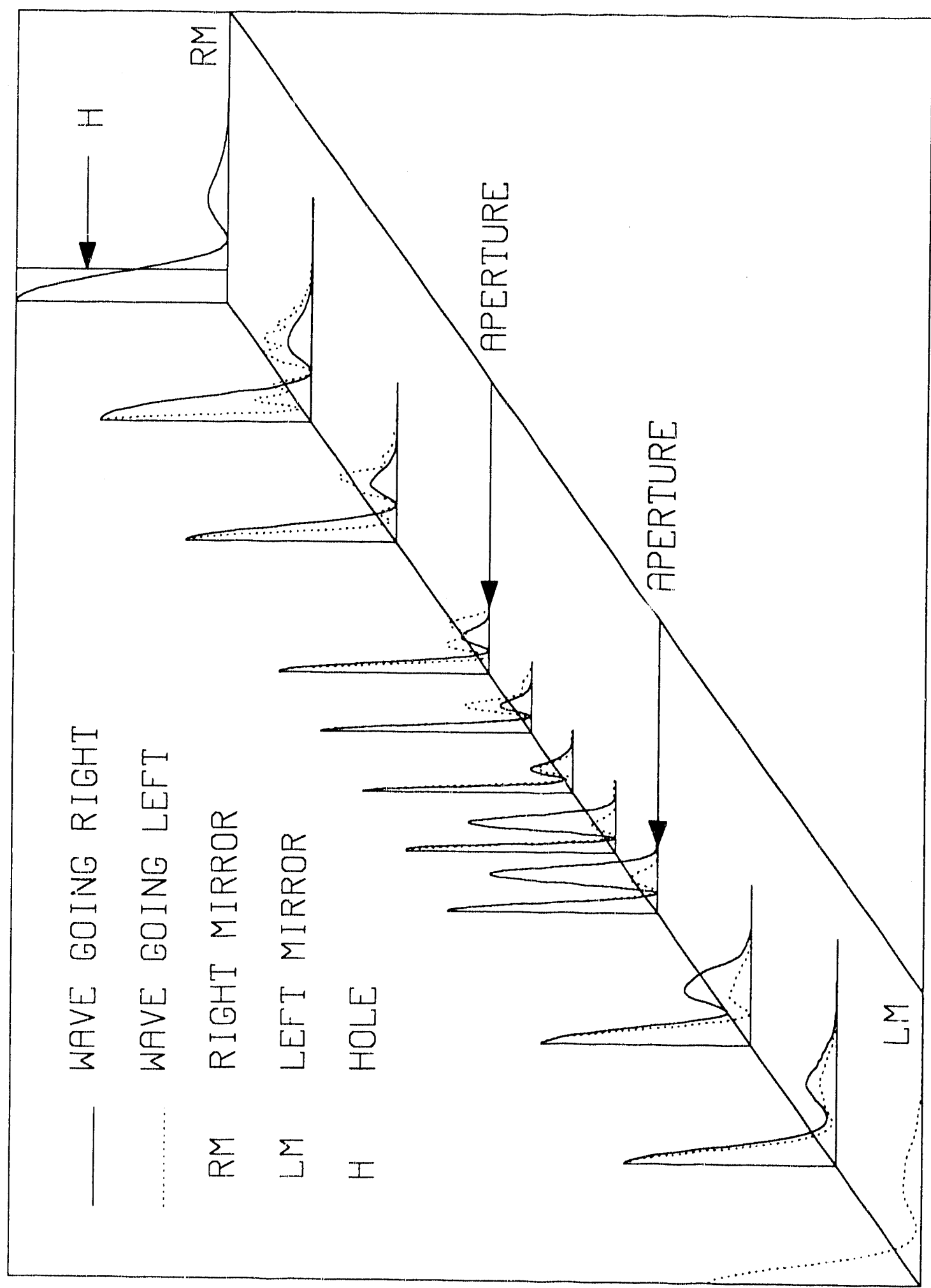




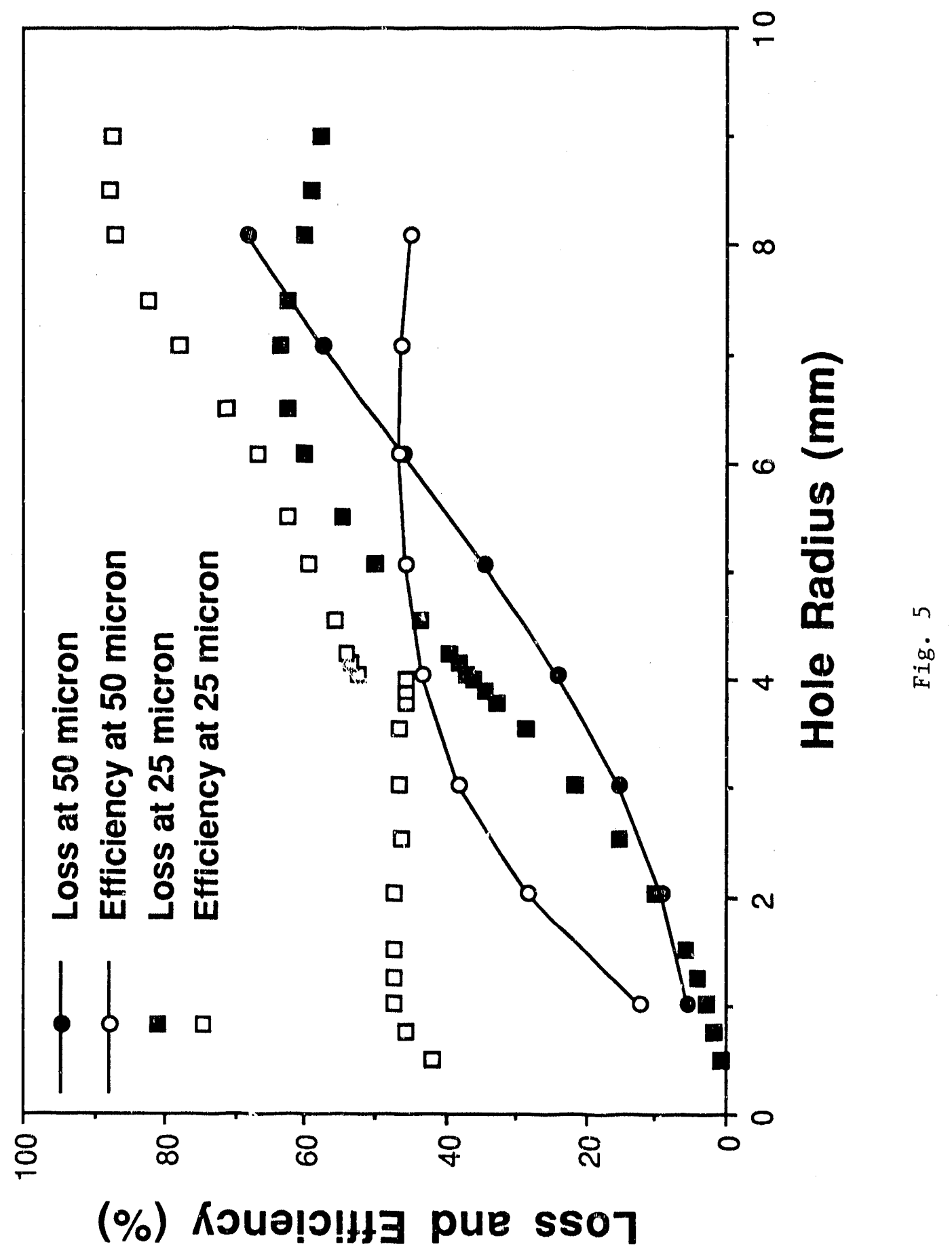




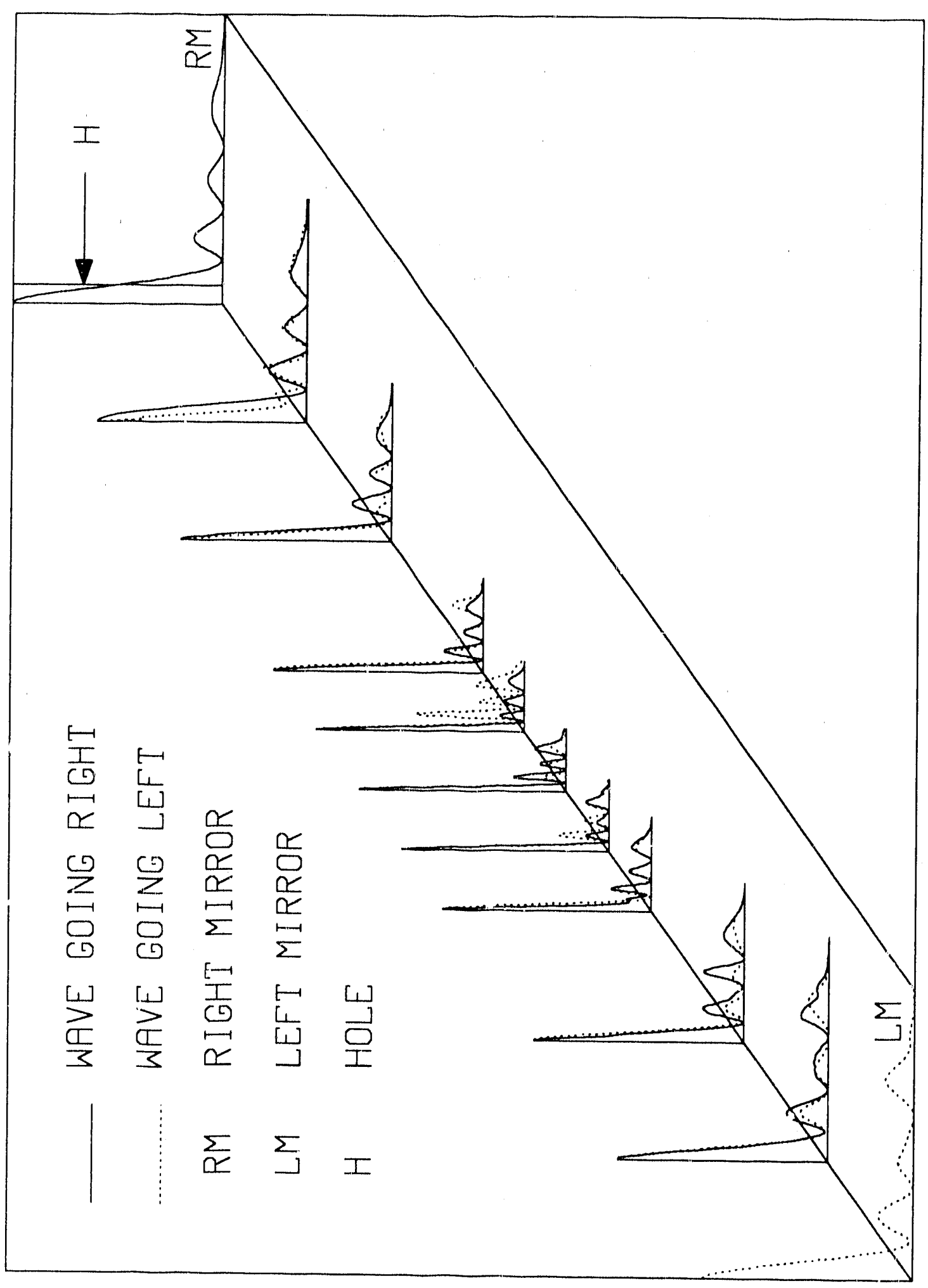

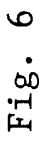



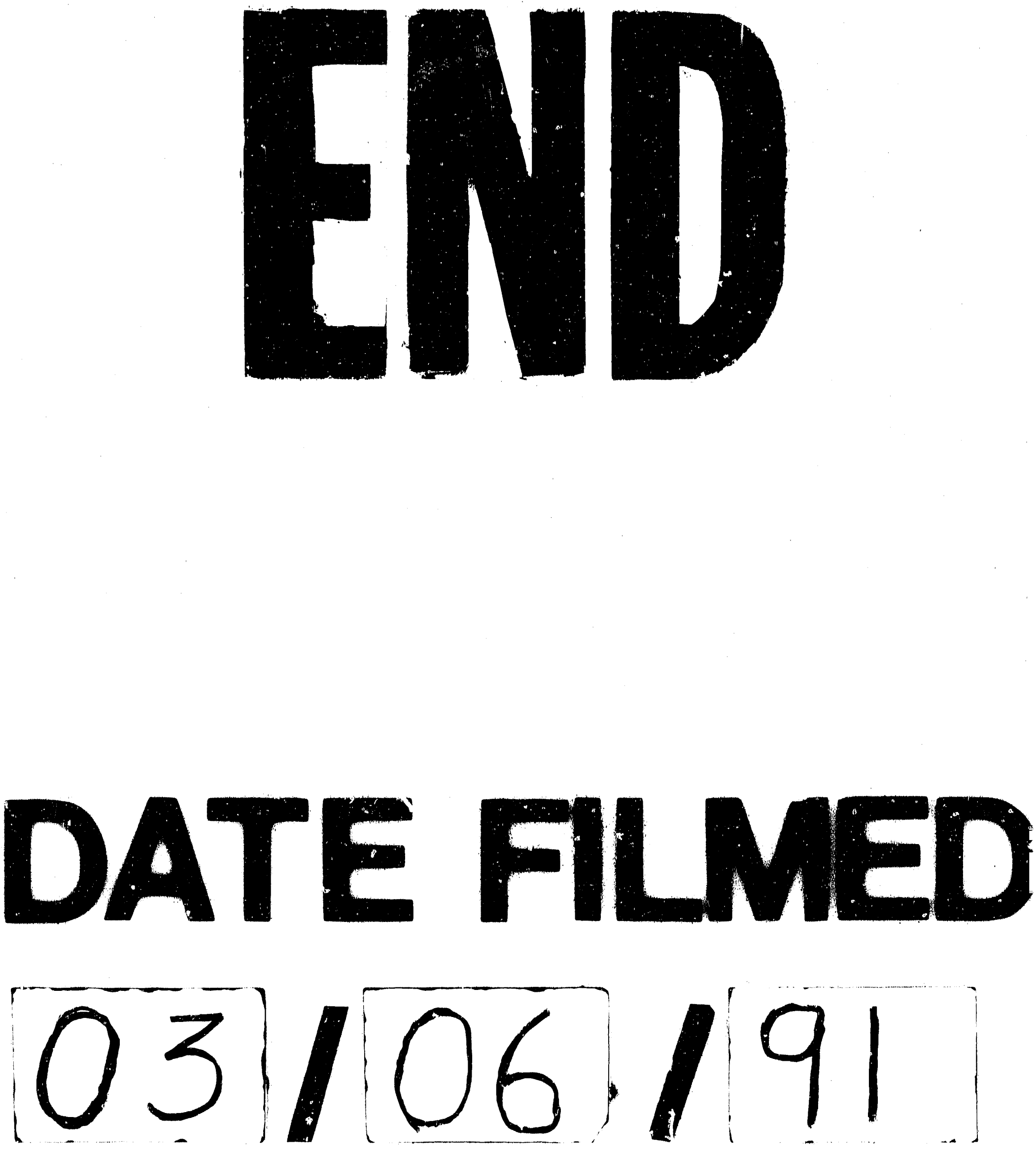
